\title{
Mixed Discretization of the Time Domain MFIE at Low Frequencies
}

\author{
H. Arda Ülkü, Member, IEEE, Ignace Bogaert, Kristof Cools, Francesco P. Andriulli, Senior Member, IEEE, \\ and Hakan Bağc1, Senior Member, IEEE
}

\begin{abstract}
Solution of the magnetic field integral equation (MFIE), which is obtained by the classical marching on-in-time (MOT) scheme, becomes inaccurate when the time step is large, i.e., under low-frequency excitation. It is shown here that the inaccuracy stems from the classical MOT scheme's failure to predict the correct scaling of the current's Helmholtz components for large time steps. A recently proposed mixed discretization strategy is used to alleviate the inaccuracy problem by restoring the correct scaling of the current's Helmholtz components under low-frequency excitation.
\end{abstract}

Index Terms-Marching on-in-time (MOT) method, magnetic field integral equation (MFIE), transient analysis, low-frequency analysis, Buffa-Christiansen functions, mixed discretization.

\section{INTRODUCTION}

$\mathbf{T}$ HE classical marching on-in-time (MOT) scheme developed for solving time-domain electric and magnetic field integral equations (TD-EFIE and TD-MFIE) use Rao-WiltonGlisson (RWG) and polynomial basis functions to expand the current in space and time, respectively. This expansion is inserted into the TD-EFIE and TD-MFIE and the resulting equations are tested with RWG functions at discrete times. The conditioning and accuracy of this classical MOT-TDEFIE solver at low frequencies, i.e., when the scatterer size is comparable to $c \Delta t$, have been studied extensively [1]-[4]. Here, $c$ is the speed of light and $\Delta t$ is the time step. Under this condition, the MOT-TD-EFIE matrix system becomes illconditioned and cannot be solved using iterative schemes. This problem, which is known as the "low-frequency breakdown" of the EFIE, stems from the fact that the Helmholtz decomposed MOT-TD-EFIE matrix is not balanced in scaling with $\Delta t$ as $\Delta t \rightarrow \infty$ and can be remedied with loop/star [1] and hierarchical preconditioning techniques [2]-[4].

On the other hand, behavior of the MOT-TD-MFIE matrix system as $\Delta t \rightarrow \infty$ has never been investigated. This work, for the first time, studies this behavior. Its contribution is twofold: (i) It rigorously shows that the solution of the classical

H. A. Ülkü was with the Division of Computer, Electrical, and Mathematical Science and Engineering, KAUST, Thuwal, 23955-6900, Saudi Arabia. $\mathrm{He}$ is now with the Department of Electronics Engineering, Gebze Technical University, Kocaeli, Turkey.

I. Bogaert was with the Department of Information Technology, Ghent University, Ghent, Belgium. He is now with the ArcelorMittal, Ghent. Belgium.

K. Cools is with the Department of Electrical and Electronic Engineering, University of Nottingham, Nottingham, UK.

F. P. Andriulli is with the Microwave Department, TELECOM Bretagne, Brest, France.

H. Bağc1 is with the Division of Computer, Electrical, and Mathematical Science and Engineering, KAUST, Thuwal, 23955-6900, Saudi Arabia (email: hakan.bagci@kaust.edu.sa).

Manuscript received June 22, 2016.
MOT-TD-MFIE matrix system does not scale correctly in $\Delta t$ as $\Delta t \rightarrow \infty$. The non-solenoidal component of the current scales as $O(1)$, which does not yield a finite value for the charge when integrated in time. Consequently, the accuracy of the solution deteriorates regardless of the integration rule used for computing the MOT matrix entries. (ii) It shows that the mixed discretization scheme, which has been originally developed in [5] for solving the frequency domain MFIE, restores the correct scaling, i.e., the non-solenoidal component of the current scales as $O\left(\Delta t^{-1}\right)$. Consequently, the solution of the mixed-discretized TD-MFIE maintains its accuracy for large $\Delta t$. This is also shown by numerical results.

Mixed discretization [5] uses divergence conforming RWG and curl conforming Buffa-Christiansen (BC) functions [6], i.e., $\hat{n} \times B C$, as basis and testing functions. Unlike the classical discretization, this scheme conforms with respect to the function spaces of the MFIE operator's both domain and range [5][8] and preserves the correct frequency scaling of the solution's Helmholtz components [9], [10]. As a result, solution of the mixed-discretized MFIE is more accurate than the classicallydiscretized MFIE especially at low frequencies [5]-[10].

\section{FORMULATION}

\section{A. TD-MFIE and MOT Scheme}

Let $S$ represent the surface of a perfect electric conductor residing in an unbounded homogeneous background medium. A magnetic field $\mathbf{H}^{\mathrm{i}}(\mathbf{r}, t)$ bandlimited to $f_{\max }$ is incident on the conductor. Enforcing the boundary condition on the total magnetic field on $S$ yields the TD-MFIE [11], [12]:

$\hat{\mathbf{n}}(\mathbf{r}) \times \mathbf{H}^{\mathrm{i}}(\mathbf{r}, t)=\frac{1}{2} \mathbf{J}(\mathbf{r}, t)-\hat{\mathbf{n}}(\mathbf{r}) \times \nabla \times \int_{S} \frac{\mathbf{J}\left(\mathbf{r}^{\prime}, t-R / c\right)}{4 \pi R} d \mathbf{r}^{\prime}$.

Here, $R=\left|\mathbf{r}-\mathbf{r}^{\prime}\right|$ is the distance between observer and source points, $\mathbf{r}$ and $\mathbf{r}^{\prime}$, and $\hat{\mathbf{n}}(\mathbf{r})$ is the outward pointing unit normal vector on $S$. To numerically solve (1), $\mathbf{J}(\mathbf{r}, t)$ is approximated using spatial and temporal basis functions, $\mathbf{f}_{n}(\mathbf{r})$ and $T_{i}(t)$ :

$$
\mathbf{J}(\mathbf{r}, t)=\sum_{n=1}^{N} \sum_{i=1}^{N_{\mathrm{t}}} I_{i, n} T_{i}(t) \mathbf{f}_{n}(\mathbf{r}) .
$$

Here, $\mathbf{f}_{n}(\mathbf{r})$ are divergence conforming RWG functions, $T_{i}(t)=T(t-i \Delta t)$, where $T(t)$ is first order piecewise polynomial Lagrange interpolation function [11], [12], and $I_{i, n}$ are the unknown current coefficients. Inserting (2) into (1) and testing the resulting equation by $\mathbf{t}_{m}(\mathbf{r}), m=1: N$, at times $t=j \Delta t$ yield the MOT matrix system [11], [12]:

$$
\mathbf{Z}_{0} \mathbf{I}_{j}=\mathbf{V}_{j}-\sum_{i=0}^{j-1} \mathbf{Z}_{j-i} \mathbf{I}_{i}, \quad j=1: N_{\mathrm{t}} .
$$


Here, $\left\{\mathbf{I}_{i}\right\}_{n}=I_{i, n},\left\{\mathbf{V}_{j}\right\}_{m}=V_{j}\left\{\mathbf{t}_{m}\right\}$, and $\left\{\mathbf{Z}_{k}\right\}_{m, n}=$ $Z_{k}\left\{\mathbf{t}_{m}, \mathbf{f}_{n}\right\}$, where the operators $V_{j}\left\{\mathbf{t}_{m}\right\}, G_{k}\left\{\mathbf{t}_{m}, \mathbf{f}_{n}\right\}$, $K_{k}\left\{\mathbf{t}_{m}, \mathbf{f}_{n}\right\}, Z_{k}\left\{\mathbf{t}_{m}, \mathbf{f}_{n}\right\}$ are defined as

$$
\begin{gathered}
V_{j}\left\{\mathbf{t}_{m}\right\}=\int_{S_{m}} \mathbf{t}_{m}(\mathbf{r}) \cdot \hat{\mathbf{n}} \times \mathbf{H}^{\mathrm{i}}(\mathbf{r}, j \Delta t) d \mathbf{r} \\
Z_{k}\left\{\mathbf{t}_{m}, \mathbf{f}_{n}\right\}=\frac{1}{2} G_{k}\left\{\mathbf{t}_{m}, \mathbf{f}_{n}\right\}-\frac{1}{4 \pi} K_{k}\left\{\mathbf{t}_{m}, \mathbf{f}_{n}\right\} \\
G_{k}\left\{\mathbf{t}_{m}, \mathbf{f}_{n}\right\}=\int_{S_{m}} \mathbf{t}_{m}(\mathbf{r}) \cdot \mathbf{f}_{n}(\mathbf{r}) T(k \Delta t) d \mathbf{r} \\
K_{k}\left\{\mathbf{t}_{m}, \mathbf{f}_{n}\right\}= \\
\int_{S_{m}} \mathbf{t}_{m}(\mathbf{r}) \cdot \hat{\mathbf{n}}(\mathbf{r}) \times \int_{S_{n}} \nabla\left[\frac{T(k \Delta t-R / c)}{R}\right] \times \mathbf{f}_{n}\left(\mathbf{r}^{\prime}\right) d \mathbf{r}^{\prime} d \mathbf{r} .
\end{gathered}
$$

The choice of $\mathbf{t}_{m}(\mathbf{r})$ determines the spatial discretization schemes termed "classical" and "mixed" as detailed in Sections II-B and II-C, respectively. $\Delta t$ depends only on $f_{\max }$ : $\Delta t=1 /\left(\alpha f_{\max }\right)$, where $\alpha$ is the over-sampling factor and $5 \leq \alpha \leq 20$. For a given spatial discretization, choosing a high value for $\alpha$ increases $N_{\mathrm{t}}$ unnecessarily without any additional gain in accuracy. When $f_{\max }$ is small, $\Delta t$ should ideally be chosen large.

\section{B. Classical Discretization}

It has been shown in [5] and [8] that to obtain accurate results, the discretization of an integral equation should be conforming with respect to the function spaces, where the range and domain of the integral operator reside, and the resulting matrix system should be well-conditioned. For the MFIE, conforming discretization means that the testing functions $\mathbf{t}_{m}(\mathbf{r})$ should reside in the dual space of the divergence conforming RWG basis functions $\mathbf{f}_{n}(\mathbf{r})$. Curl conforming RWG testing functions $\mathbf{t}_{m}(\mathbf{r})=\hat{\mathbf{n}}(\mathbf{r}) \times \mathbf{f}_{m}(\mathbf{r})$ satisfy this condition. However, the resulting Gram matrix with entries $G_{0}\left\{\hat{\mathbf{n}} \times \mathbf{f}_{m}, \mathbf{f}_{n}\right\}$ is singular, which makes the solution of (3) impossible. Therefore, in the literature, the choice $\mathbf{t}_{m}(\mathbf{r})=$ $\mathbf{f}_{m}(\mathbf{r})$ is adopted. In this work the scheme resulting from this choice of testing function is termed the classical discretization scheme. In what follows here, it is rigorously shown that the current obtained by solving the classically-discretized TDMFIE has incorrect scaling in $\Delta t$ under plane-wave excitation. It should be noted here that the results of the analysis carried out in Sections II-B and II-C are valid for any excitation that can be represented by a plane-wave expansion.

The behavior of the Helmholtz components of the current as $\Delta t \rightarrow \infty$ can be analyzed by decomposing the RWG space into two components spanned by loop and star functions. Assume that $\mathbf{J}(\mathbf{r}, t)$ is approximated as

$$
\begin{aligned}
\mathbf{J}(\mathbf{r}, t) & =\sum_{n=1}^{N_{\mathrm{l}}} \sum_{i=1}^{N_{\mathrm{t}}} I_{i, n}^{\mathrm{l}} T_{i}(t) \mathbf{f}_{n}^{\mathrm{l}}(\mathbf{r}) \\
& +\sum_{n=1}^{N_{\mathrm{s}}} \sum_{i=1}^{N_{\mathrm{t}}} I_{i, n}^{\mathrm{s}} T_{i}(t) \mathbf{f}_{n}^{\mathrm{s}}(\mathbf{r}) .
\end{aligned}
$$

Here, $N_{\mathrm{l}}+N_{\mathrm{s}}=N$ and $\mathbf{f}_{n}^{\mathrm{l}}(\mathbf{r})$ and $\mathbf{f}_{n}^{\mathrm{s}}(\mathbf{r})$ are loop and star basis functions, which are constructed from linear combinations of RWG functions [13]. Inserting (8) into (2) and testing the resulting equation with $\mathbf{f}_{m}^{\mathrm{l}}(\mathbf{r}), m=1: N_{\mathrm{l}}$ and $\mathbf{f}_{m}^{\mathrm{s}}(\mathbf{r})$, $m=1: N_{\mathrm{s}}$, at times $t=j \Delta t$ yield

$$
\mathbf{Z}_{0}^{\mathrm{ls}} \mathbf{I}_{j}^{\mathrm{ls}}=\mathbf{V}_{j}^{\mathrm{ls}}-\sum_{i=0}^{j-1} \mathbf{Z}_{j-i}^{\mathrm{ls}} \mathbf{I}_{i}^{\mathrm{ls}}, \quad j=1: N_{\mathrm{t}} .
$$

Here, the entries of the blocks of the matrix $\mathbf{Z}_{k}^{\text {ls }}$ and the vectors $\mathbf{V}_{i}^{\mathrm{ls}}$ and $\mathbf{I}_{i}^{\mathrm{ls}}$ are given by

$$
\begin{gathered}
\left\{\mathbf{Z}_{k}^{\mathrm{ls}}\right\}_{m, n}=\left\{\begin{array}{l}
Z_{k}\left\{\mathbf{f}_{m}^{\mathrm{l}}, \mathbf{f}_{n}^{\mathrm{l}}\right\}, m=1: N_{\mathrm{l}}, n=1: N_{\mathrm{l}} \\
Z_{k}\left\{\mathbf{f}_{m-N_{\mathrm{l}}}^{\mathrm{s}}, \mathbf{f}_{n}^{\mathrm{l}}\right\}, m=N_{\mathrm{l}}+1: N, n=1: N_{\mathrm{l}} \\
Z_{k}\left\{\mathbf{f}_{m}^{\mathrm{l}}, \mathbf{f}_{n-N_{1}}^{\mathrm{s}}\right\}, m=1: N, n=N_{\mathrm{l}}+1: N \\
Z_{k}\left\{\mathbf{f}_{m-N_{1}}^{\mathrm{s}}, \mathbf{f}_{n-N_{\mathrm{l}}}^{\mathrm{s}}\right\}, N_{\mathrm{l}}+1: N, n=N_{\mathrm{l}}+1: N
\end{array}\right. \\
\left\{\mathbf{V}_{j}^{\mathrm{ls}}\right\}_{m}=\left\{\begin{array}{l}
V_{j}\left\{\mathbf{f}_{m}^{\mathrm{l}}\right\}, m=1: N_{\mathrm{l}} \\
V_{j}\left\{\mathbf{f}_{m-N_{\mathrm{l}}}^{\mathrm{s}}\right\}, m=N_{\mathrm{l}}+1: N
\end{array}\right. \\
\left\{\mathbf{I}_{i}^{\mathrm{ls}}\right\}_{n}=\left\{\begin{array}{l}
\left\{\mathbf{I}_{i}^{\mathrm{l}}\right\}_{n}=I_{i, n}^{1}, n=1: N_{\mathrm{l}} \\
\left\{\mathbf{I}_{i}^{\mathrm{s}}\right\}_{n-N_{\mathrm{l}}}=I_{i, n-N_{\mathrm{l}}}^{\mathrm{s}}, n=N_{\mathrm{l}}+1: N
\end{array}\right.
\end{gathered}
$$

The scaling of $\mathbf{I}_{j}^{\mathrm{ls}}$ as $\Delta t \rightarrow \infty$ can be obtained from the scaling of $\mathbf{Z}_{0}^{\mathrm{ls}}$ and $\mathbf{V}_{j}^{\mathrm{ls}}$. It is obvious that $G_{0}\left\{\mathbf{f}_{m}^{\mathrm{p}}, \mathbf{f}_{n}^{\mathrm{q}}\right\} \sim O(1), \mathrm{p}, \mathrm{q} \in$ $\{1, \mathrm{~s}\}$. Consider the Taylor series expansion of the gradient term in (7) with $k=0$ :

$$
\begin{gathered}
\nabla\left[\frac{T(-R / c)}{R}\right]=\nabla\left[\sum_{p=0}^{\infty} \frac{(-1)^{p} T^{(p)}(0) R^{p-1}}{p ! c^{p}}\right] \\
=T(0) \nabla R^{-1}+\hat{\mathbf{R}} \sum_{p=2}^{\infty} \frac{(-1)^{p} T^{(p)}(0) R^{p-2}}{p ! c^{p}} .
\end{gathered}
$$

Here, $T^{(p)}(0)=\left.\partial_{t}^{p} T(t)\right|_{t=0}$ and $\hat{\mathbf{R}}=\left(\mathbf{r}-\mathbf{r}^{\prime}\right) / R$. The first term in the right hand side (RHS) of (11) scales as $O(1)$. The terms in the summation scale as $O\left(\Delta t^{-p}\right), p \geq 2$, due to the derivatives $T^{(p)}(0)$. By inserting (11) into the expression of $K_{0}\left\{\mathbf{f}_{m}^{\mathrm{p}}, \mathbf{f}_{n}^{\mathrm{q}}\right\}, \mathrm{p}, \mathrm{q} \in\{1, \mathrm{~s}\}$, one can show that, its dominant terms all scale as $O(1)$. From expressions of $V_{j}\left\{\mathbf{f}_{m}^{l}\right\}$ and $V_{j}\left\{\mathbf{f}_{m}^{\mathrm{s}}\right\}$, it can be concluded that $V_{j}\left\{\mathbf{f}_{m}^{\mathrm{l}}\right\}$ and $V_{j}\left\{\mathbf{f}_{m}^{\mathrm{s}}\right\}$ scale as $O(1)$. Consequently, one obtains

$$
\mathbf{Z}_{0}^{\mathrm{ls}} \sim\left[\begin{array}{ll}
O(1) & O(1) \\
O(1) & O(1)
\end{array}\right], \mathbf{V}_{j}^{\mathrm{ls}} \sim\left[\begin{array}{l}
O(1) \\
O(1)
\end{array}\right], \mathbf{I}_{i}^{\mathrm{ls}} \sim\left[\begin{array}{l}
O(1) \\
O(1)
\end{array}\right] .
$$

Inserting (8) into the equation of continuity $\rho(\mathbf{r}, t)=$ $-\int_{0}^{t} \nabla \cdot \mathbf{J}(\mathbf{r}, \tau) d \tau$ and using the fact that $\nabla \cdot \mathbf{f}_{n}^{\mathrm{l}}(\mathbf{r})=0$, one can obtain

$$
\rho(\mathbf{r}, t)=-\sum_{n=1}^{N_{\mathrm{s}}} \sum_{i=1}^{N_{\mathrm{t}}} I_{i, n}^{\mathrm{s}} \nabla \cdot \mathbf{f}_{n}^{\mathrm{s}}(\mathbf{r}) \int_{0}^{t} T_{i}(\tau) d \tau .
$$

Here, $\rho(\mathbf{r}, t)$ is the charge density. The integral in (13) scales as $O(\Delta t)$. Therefore, $\mathbf{I}_{i}^{\mathrm{s}}$ has to scale as $O\left(\Delta t^{-1}\right)$ to obtain a finite value for $\rho(\mathbf{r}, t)$. This immediately shows that $\mathbf{I}_{i}^{\mathbf{s}}$ obtained by solving (9) does not scale correctly as $\Delta t \rightarrow \infty$.

\section{Mixed Discretization}

Mixed discretization scheme uses the rotated $\mathrm{BC}$ functions as testing functions, i.e., $\mathbf{t}_{m}(\mathbf{r})=\hat{\mathbf{n}}(\mathbf{r}) \times \mathbf{g}_{m}(\mathbf{r}), m=1: N$, where $\mathbf{g}_{m}(\mathbf{r})$ denote the divergence conforming BC functions [6]. Since $\hat{\mathbf{n}}(\mathbf{r}) \times \mathbf{g}_{m}(\mathbf{r})$ are curl conforming, the mixed discretization is conforming with respect to the function space of the MFIE operator's range. Additionally, the resulting Gram matrix with entries $G_{0}\left\{\hat{\mathbf{n}} \times \mathbf{g}_{m}, \mathbf{f}_{n}\right\}$ is well-conditioned [14]. In what follows here, it is shown that the current obtained by 


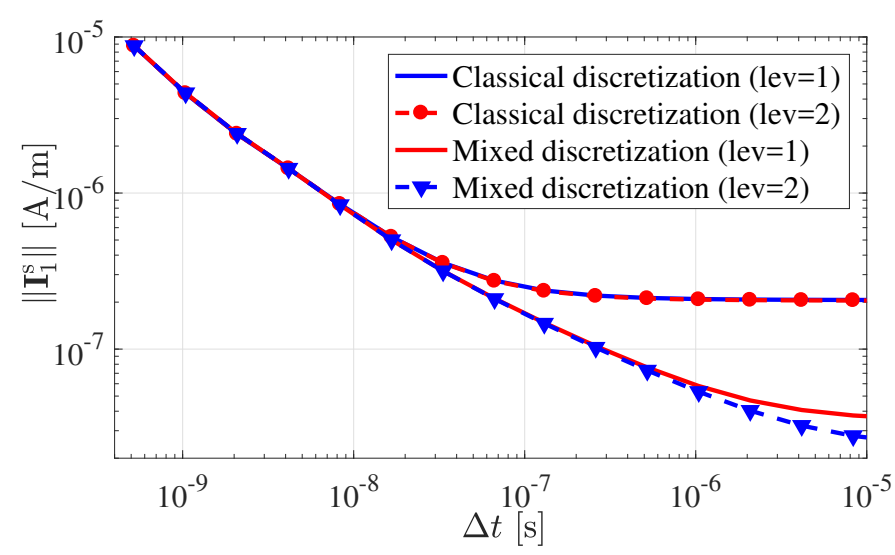

Fig. 1. $L_{2}$-norm of the star coefficients at the first time step, $t=\Delta t$.

solving the mixed-discretized TD-MFIE has the correct scaling in $\Delta t$ under plane-wave excitation.

The behavior of $\mathbf{I}_{i}^{\mathrm{ls}}$ as $\Delta t \rightarrow \infty$ is analyzed by decomposing the RWG space into two subspaces spanned $\mathbf{f}_{n}^{\mathrm{l}}(\mathbf{r})$ and $\mathbf{f}_{n}^{\mathrm{s}}(\mathbf{r})$, respectively. Expansion (8) is inserted into (1) and the resulting equation is tested with loop and star functions generated from linear combinations of BC functions, $\hat{\mathbf{n}}(\mathbf{r}) \times \mathbf{g}_{m}^{1}(\mathbf{r}), m=1$ : $N_{\mathrm{l}}$ and $\hat{\mathbf{n}}(\mathbf{r}) \times \mathbf{g}_{m}^{\mathrm{s}}(\mathbf{r}), m=1: N_{\mathrm{s}}$, at times $t=j \Delta t$. The resulting linear MOT system reads

$$
\tilde{\mathbf{Z}}_{0}^{\mathrm{ls}} \mathbf{I}_{j}^{\mathrm{ls}}=\tilde{\mathbf{V}}_{j}^{\mathrm{ls}}-\sum_{i=0}^{j-1} \tilde{\mathbf{Z}}_{j-i}^{\mathrm{ls}} \mathbf{I}_{i}^{\mathrm{ls}}, \quad j=1: N_{\mathrm{t}} .
$$

The entries of the matrix $\tilde{\mathbf{Z}}_{k}^{\text {ls }}$ and the vector $\tilde{\mathbf{V}}_{j}^{\text {ls }}$ are given by the expressions obtained by replacing $\mathbf{f}_{m}^{\mathrm{l}}$ and $\mathbf{f}_{m}^{\mathrm{s}}$ with $\hat{\mathbf{n}} \times$ $\mathbf{g}_{m}^{\mathrm{l}}$ and $\hat{\mathbf{n}} \times \mathbf{g}_{m}^{\mathrm{s}}$, in (10) respectively. The scaling of $\mathbf{I}_{j}^{\mathrm{ls}}$ as $\Delta t \rightarrow \infty$ can be obtained from the scaling of $\tilde{\mathbf{Z}}_{0}^{\text {ls }}$ and $\tilde{\mathbf{V}}_{j}^{\text {ls }}$. The dominant terms in $Z_{0}\left\{\hat{\mathbf{n}} \times \mathbf{g}_{m}^{\mathrm{l}}, \mathbf{f}_{n}^{\mathrm{s}}\right\}, Z_{0}\left\{\hat{\mathbf{n}} \times \mathbf{g}_{m}^{\mathbf{s}}, \mathbf{f}_{n}^{\mathrm{l}}\right\}$, and $Z_{0}\left\{\hat{\mathbf{n}} \times \mathbf{g}_{m}^{\mathrm{s}}, \mathbf{f}_{n}^{\mathrm{s}}\right\}$ come from $G_{0}\left\{\hat{\mathbf{n}} \times \mathbf{g}_{m}^{\mathrm{l}}, \mathbf{f}_{n}^{\mathrm{s}}\right\}, G_{0}\left\{\hat{\mathbf{n}} \times \mathbf{g}_{m}^{\mathrm{s}}, \mathbf{f}_{n}^{\mathrm{l}}\right\}$, and $G_{0}\left\{\hat{\mathbf{n}} \times \mathbf{g}_{m}^{\mathrm{s}}, \mathbf{f}_{n}^{\mathrm{s}}\right\}$, respectively. Therefore, they all scale as $O(1)$. On the other hand, analysis of $Z_{0}\left\{\hat{\mathbf{n}} \times \mathbf{g}_{m}^{1}, \mathbf{f}_{n}^{1}\right\}$ 's scaling requires a more detailed investigation as described next.

The RWG and BC loop functions can be represented as the surface curl of the pyramid shaped functions:

$$
\begin{aligned}
\mathbf{f}_{n}^{\mathrm{l}}(\mathbf{r}) & =\hat{\mathbf{n}}(\mathbf{r}) \times \nabla_{\mathrm{S}} \varphi_{n}(\mathbf{r}) \\
\mathbf{g}_{m}^{\mathrm{l}}(\mathbf{r}) & =\hat{\mathbf{n}}(\mathbf{r}) \times \nabla_{\mathrm{S}} \vartheta_{m}(\mathbf{r})
\end{aligned}
$$

where $\varphi_{n}(\mathbf{r}), \mathbf{r} \in S_{n}^{1}$ [10], and $\vartheta_{m}(\mathbf{r}), \mathbf{r} \in S_{m}^{1}$ [6], are the pyramid shape functions, $S_{n}^{\mathrm{l}}$ and $S_{m}^{\mathrm{l}}$, represent the union of triangular patches that support $\varphi_{n}(\mathbf{r})$ and $\vartheta_{m}(\mathbf{r})$, and $\nabla_{\mathrm{S}}$ is the surface gradient operator. It should be noted that $\varphi_{n}(\mathbf{r})$ and $\vartheta_{m}(\mathbf{r})$ are continuous on triangular patches and vanish at the boundaries of $S_{n}^{\mathrm{l}}$ and $S_{m}^{\mathrm{l}}$. Inserting (15) and (16) into $G_{0}\left\{\hat{\mathbf{n}} \times \mathbf{g}_{m}^{\mathrm{l}}, \mathbf{f}_{n}^{\mathrm{l}}\right\}$ and applying surface divergence theorem to the resulting equation yield:

$$
\begin{aligned}
& G_{0}\left\{\hat{\mathbf{n}} \times \mathbf{g}_{m}^{1}, \mathbf{f}_{n}^{\mathrm{l}}\right\}=\int_{S_{m}^{1}} \vartheta_{m}(\mathbf{r}) \hat{\mathbf{n}}(\mathbf{r}) \cdot\left[\nabla_{\mathrm{S}} \times \nabla_{\mathrm{S}} \varphi_{n}(\mathbf{r})\right] d \mathbf{r} \\
& -\int_{\partial S_{m}^{1}} \vartheta_{m}(\mathbf{r}) \hat{\mathbf{m}}(\mathbf{r}) \cdot\left[\hat{\mathbf{n}}(\mathbf{r}) \times \nabla_{\mathrm{S}} \varphi_{n}(\mathbf{r})\right] d \mathbf{r}
\end{aligned}
$$

Here, $\hat{\mathbf{m}}(\mathbf{r})$ is the outward pointing unit normal vector of $\partial S_{m}^{\mathrm{l}}$, the boundary of $S_{m}^{\mathrm{l}}$. Since $\vartheta_{m}(\mathbf{r})=0$ for $\mathbf{r} \in \partial S_{m}^{\mathrm{l}}$ and
$\nabla_{\mathrm{S}} \times \nabla_{\mathrm{S}} \varphi_{n}(\mathbf{r})=0$, then $G_{0}\left\{\hat{\mathbf{n}} \times \mathbf{g}_{m}^{\mathrm{l}}, \mathbf{f}_{n}^{\mathrm{l}}\right\}=0$ and $Z_{0}\{\hat{\mathbf{n}} \times$ $\left.\mathbf{g}_{m}^{\mathrm{l}}, \mathbf{f}_{n}^{\mathrm{l}}\right\}=-K_{0}\left\{\hat{\mathbf{n}} \times \mathbf{g}_{m}^{\mathrm{l}}, \mathbf{f}_{n}^{\mathrm{l}}\right\} /(4 \pi)$. Inserting (10) into the expression of $K_{0}\left\{\hat{\mathbf{n}} \times \mathbf{g}_{m}^{1}, \mathbf{f}_{n}^{1}\right\}$ yields:

$$
\begin{aligned}
& K_{0}\left\{\hat{\mathbf{n}} \times \mathbf{g}_{m}^{\mathrm{l}}, \mathbf{f}_{n}^{\mathrm{l}}\right\}=\int_{S_{m}} \mathbf{g}_{m}^{\mathrm{l}}(\mathbf{r}) \cdot \int_{S_{n}} \nabla R^{-1} \times \mathbf{f}_{n}^{\mathrm{l}}\left(\mathbf{r}^{\prime}\right) d \mathbf{r}^{\prime} d \mathbf{r} \\
+ & \int_{S_{m}} \mathbf{g}_{m}^{\mathrm{l}}(\mathbf{r}) \cdot \int_{S_{n}} \sum_{p=2}^{\infty} \frac{(-1)^{p} T^{(p)}(0) R^{p-2}}{p ! c^{p}} \hat{\mathbf{R}} \times \mathbf{f}_{n}^{\mathrm{l}}\left(\mathbf{r}^{\prime}\right) d \mathbf{r}^{\prime} d \mathbf{r} .
\end{aligned}
$$

The first term of the RHS in (18) represents the static magnetic field due to a loop source tested by a loop function and is zero [15]. Terms of the summation in the RHS scale as $O\left(\Delta t^{-p}\right), p \geq 2$, due to derivatives $T^{(p)}(0)$. Therefore, the dominant term in $K_{0}\left\{\hat{\mathbf{n}} \times \mathbf{g}_{m}^{l}, \mathbf{f}_{n}^{\mathrm{l}}\right\}$ and hence $Z_{0}\left\{\hat{\mathbf{n}} \times \mathbf{g}_{m}^{\mathrm{l}}, \mathbf{f}_{n}^{\mathrm{l}}\right\}$ scales as $O\left(\Delta t^{-2}\right)$. As a result,

$$
\tilde{\mathbf{Z}}_{0}^{\text {ls }} \sim\left[\begin{array}{ll}
O\left(\Delta t^{-2}\right) & O(1) \\
O(1) & O(1)
\end{array}\right] .
$$

The scaling of $\tilde{\mathbf{V}}_{j}^{\text {ls }}$ can be determined using $V_{j}\left\{\hat{\mathbf{n}} \times \mathbf{g}_{m}^{\mathrm{l}}\right\}$ and $V_{j}\left\{\hat{\mathbf{n}} \times \mathbf{g}_{m}^{\mathrm{s}}\right\}$. It can be easily seen that $V_{j}\left\{\hat{\mathbf{n}} \times \mathbf{g}_{m}^{\mathrm{s}}\right\} \sim O(1)$. Inserting (16) into the expression of $V_{j}\left\{\hat{\mathbf{n}} \times \mathbf{g}_{m}^{1}\right\}$ and applying the chain rule and several vector manipulations to the resulting equation yield:

$$
\begin{aligned}
& V_{j}\left\{\hat{\mathbf{n}} \times \mathbf{g}_{m}^{1}\right\}=-\int_{S_{m}^{1}} \nabla_{\mathrm{S}} \cdot\left[\vartheta_{m}(\mathbf{r}) \hat{\mathbf{n}}(\mathbf{r}) \times \mathbf{H}^{\mathrm{i}}\left(\mathbf{r}, t_{j}\right)\right] d \mathbf{r} \\
& -\int_{S_{m}^{1}} \vartheta_{m}(\mathbf{r}) \hat{\mathbf{n}}(\mathbf{r}) \cdot\left[\nabla \times \mathbf{H}^{\mathrm{i}}\left(\mathbf{r}, t_{j}\right)\right] d \mathbf{r} .
\end{aligned}
$$

Surface divergence theorem is applied to the first term of the RHS. The second term is simplified assuming $\mathbf{H}^{\mathrm{i}}(\mathbf{r}, t)$ is the magnetic field of a plane wave. These operations yield:

$$
\begin{aligned}
& V_{j}\left\{\hat{\mathbf{n}} \times \mathbf{g}_{m}^{1}\right\}=-\int_{\partial S_{m}^{1}} \hat{\mathbf{m}}(\mathbf{r}) \cdot\left[\vartheta_{m}(\mathbf{r}) \hat{\mathbf{n}}(\mathbf{r}) \times \mathbf{H}^{\mathrm{i}}\left(\mathbf{r}, t_{j}\right)\right] d \mathbf{r} \\
& +\int_{S_{m}^{1}} c^{-1}(\hat{\mathbf{n}}(\mathbf{r}) \times \hat{\mathbf{k}}) \cdot \vartheta_{m}(\mathbf{r}) \partial_{t} \mathbf{H}^{\mathrm{i}}(\mathbf{r}, t) d \mathbf{r}
\end{aligned}
$$

where $\hat{\mathbf{k}}$ is the plane wave's direction of propagation. First integral in (21) is zero since $\vartheta_{m}(\mathbf{r})=0$ for $\mathbf{r} \in \partial S_{m}^{\mathrm{l}}$. Derivative in the second term indicates that $V_{j}\left\{\hat{\mathbf{n}} \times \mathbf{g}_{m}^{1}\right\} \sim O\left(\Delta t^{-1}\right)$. Consequently, one can obtain

$$
\tilde{\mathbf{V}}_{j}^{\mathrm{ls}} \sim\left[\begin{array}{c}
O\left(\Delta t^{-1}\right) \\
O(1)
\end{array}\right], \mathbf{I}_{j}^{\mathrm{ls}} \sim\left[\begin{array}{c}
O(1) \\
O\left(\Delta t^{-1}\right)
\end{array}\right] .
$$

Indeed, the scaling of $\mathbf{I}_{j}^{\mathrm{s}}$ obtained by solving (14) matches that predicted by the continuity equation as $\Delta t \rightarrow \infty$.

\section{NumericAl RESUlts}

In this section, the MOT-TD-MFIE solver, which uses classical and mixed discretization schemes, is applied to the characterization of transient scattering from a unit sphere that resides in free space and is centered at the origin. Retarded-time source integrals and the test integrals in the MOT matrix entries in (7) are computed using the semianalytical integration scheme described in [11], [12] and the Gauss-Legendre quadrature rule, respectively. Two levels of numerical integration are used: (i) "lev=1" uses seven quadrature points. (ii) "lev=2" first divides the triangles into four and uses seven quadrature points in each sub-triangle. The 


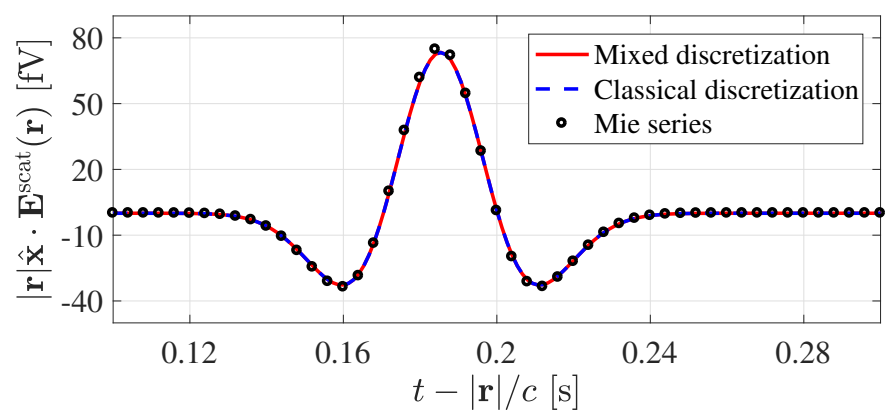

(a)

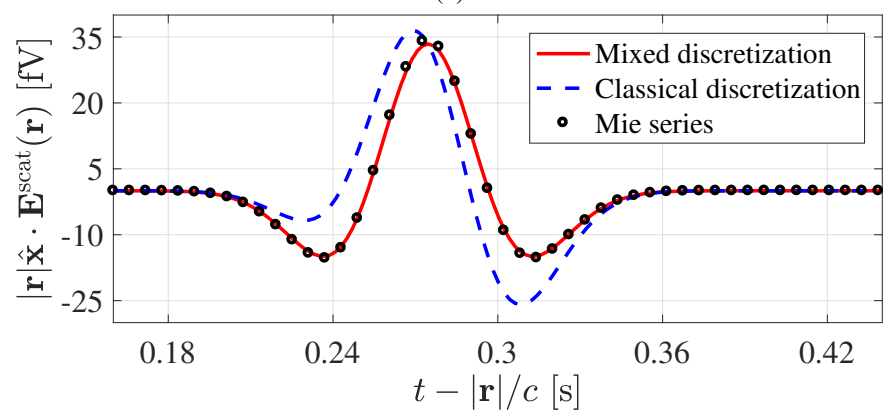

(b)

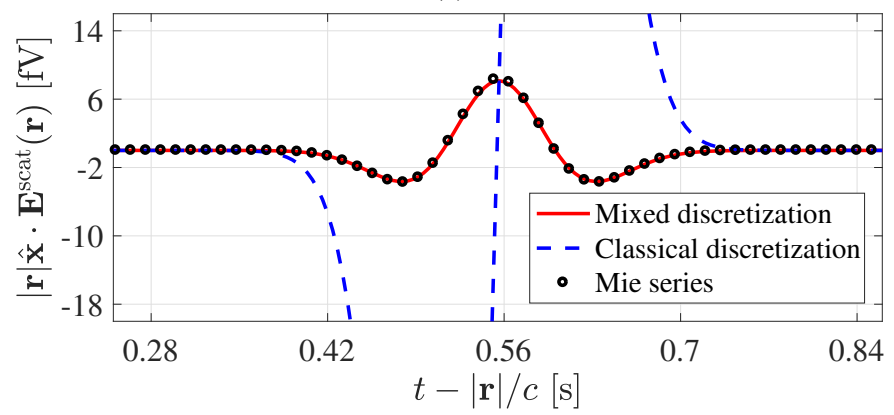

(c)

Fig. 2. The $x$-component of the range-corrected scattered electric field, $|\mathbf{r}| \hat{\mathbf{x}} \cdot \mathbf{E}^{\mathrm{scat}}(\mathbf{r})$ for (a) $\Delta t=1.33 \mathrm{~ms}$, (b) $\Delta t=2 \mathrm{~ms}$, and (c) $\Delta t=4 \mathrm{~ms}$.

excitation is a plane wave: $\mathbf{H}^{\mathrm{i}}(\mathbf{r}, t)=-\eta^{-1} \hat{\mathbf{y}} G(t+\mathbf{r} \cdot \hat{\mathbf{z}} / c)$ where $G(t)=\cos \left(2 \pi f_{0}\left[t-t_{0}\right]\right) e^{-\left(t-t_{0}\right)^{2} /\left(2 \sigma^{2}\right)}$ is a Gaussian pulse with modulation frequency $f_{0}=0.66 f_{\max }$, duration $\sigma=3.34 / f_{\max }$, and delay $t_{0}=21.7 / f_{\max }$. To investigate the scaling of $\mathbf{I}_{j}^{\text {ls }}$ as $\Delta t \rightarrow \infty, f_{\max }$ is swept in the interval [24 kHz-192 MHz], and $\Delta t=1 /\left(10 f_{\max }\right) . \mathbf{J}(\mathbf{r}, t)$ induced on the sphere is discretized using $N=750$ RWG basis functions.

Fig. 1 plots the $L_{2}$ norm of the star current coefficients at $t=\Delta t$, i.e., $\left\|\mathbf{I}_{1}^{\mathrm{s}}\right\|=\sqrt{\sum_{n=1}^{N_{\mathrm{s}}}\left|I_{1, n}^{\mathrm{s}}\right|^{2}}$, vs. $\Delta t$ as $f_{\max }$ and $\Delta t$ are swept. Note that $\mathbf{I}_{1}^{\mathbf{s}}$ are computed via loop/star decomposition after the $\mathbf{I}_{1}$ are computed by the MOT solver using the classical and mixed discretization schemes. They are not obtained by solving the MOT systems in (9) or (14). Fig. 1 clearly demonstrates that $\left\|\mathbf{I}_{1}^{\mathrm{S}}\right\|$ obtained by solving the classically-discretized TD-MFIE saturates and scales as $O(1)$ as $\Delta t$ gets larger. On the other hand, $\left\|\mathbf{I}_{1}^{\mathrm{s}}\right\|$ obtained by solving the mixed-discretized TD-MFIE scales as $O\left(\Delta t^{-1}\right)$. These results verify the analysis carried out in Sections II-B and II-C. Fig. 1 also shows that the classical discretization produces the wrong scaling regardless of the integration accuracy (due to non-conforming testing). On the other hand, higher integration accuracy helps the mixed discretization scheme achieve the correct scaling especially as $\Delta t$ gets larger.

Figs. 2(a)-(c) plot the $x$-component of the (range-corrected) scattered electric field obtained from the three sets of MOT solutions with $f_{0}=0 \mathrm{~Hz}$ and $f_{\max } \in\{75,50,25\} \mathrm{Hz}$ ( $\Delta t \in\{1.33,2,4\} \mathrm{ms})$. The figures clearly show that, as $\Delta t$ gets larger, the accuracy of the classically-discretized TDMFIE's solution deteriorates while the solution of the mixeddiscretized TD-MFIE maintains its accuracy.

\section{CONCLUSion}

The TD-MFIE discretized using RWG basis and testing functions produces inaccurate results when the $\Delta t$ is large because this discretization scheme can not predict the correct scaling of the current's Helmholtz components as $\Delta t \rightarrow \infty$. This can be avoided by using the mixed discretization scheme with RWG basis and BC testing functions that are conforming with respect to the function spaces of the MFIE operator.

\section{REFERENCES}

[1] N. W. Chen, K. Aygun, and E. Michielssen, "Integral-equation-based analysis of transient scattering and radiation from conducting bodies at very low frequencies," IET Microwaves Antennas Propag., vol. 148, no. 6, pp. 381-387, Dec. 2001.

[2] F. P. Andriulli, H. Bagci, F. Vipiana, G. Vecchi, and E. Michielssen, "A marching-on-in-time hierarchical scheme for the solution of the time domain electric field integral equation," IEEE Trans. Antennas. Propag., vol. 55, no. 12, pp. 3734-3738, Dec. 2007.

[3] _ "Analysis and regularization of the TD-EFIE low-frequency breakdown," IEEE Trans. Antennas. Propag., vol. 57, no. 7, pp. 2034-2046, July 2009.

[4] H. Bagci, F. P. Andriulli, F. Vipiana, G. Vecchi, and E. Michielssen, "A well-conditioned integral-equation formulation for efficient transient analysis of electrically small microelectronic devices," IEEE Trans. Adv. Packag., vol. 33, no. 2, pp. 468-480, May 2010.

[5] K. Cools, F. P. Andriulli, D. D. Zutter, and E. Michielssen, "Accurate and conforming mixed discretization of the MFIE," IEEE Antennas Wireless Propag. Lett., vol. 10, pp. 528-531, 2011.

[6] A. Buffa and S. H. Christiansen, "A dual finite element complex on the barycentric refinement," Math. Comput., vol. 76, pp. 1743-1769, Dec. 2007.

[7] Y. Beghein, K. Cools, H. Bagci, and D. D. Zutter, "A space-time mixed Galerkin marching-on-in-time scheme for the time-domain combined field integral equation," IEEE Trans. Antennas. Propag., vol. 61, no. 3 , pp. 1228-1238, Mar. 2013.

[8] S. H. Christiansen and J.-C. Nedelec, "A preconditioner for the electric field integral equation based on Calderon formulas," SIAM Journal on Numerical Analysis, vol. 40, no. 3, pp. 1100-1135, 2002.

[9] I. Bogaert, K. Cools, F. P. Andriulli, and H. Bagci, "Low-frequency scaling of the standard and mixed magnetic field and Müller integral equations," IEEE Trans. Antennas. Propag., vol. 62, no. 2, pp. 822-831, Feb. 2014.

[10] Y. Zhang, T. J. Cui, W. C. Chew, and J.-S. Zhao, "Magnetic field integral equation at very low frequencies," IEEE Trans. Antennas. Propag., vol. 51, no. 8, pp. 1864-1871, Aug. 2003.

[11] H. A. Ulku and A. A. Ergin, "Analytical evaluation of transient magnetic fields due to RWG current bases," IEEE Trans. Antennas. Propag., vol. 55, no. 12, pp. 3565-3575, Dec. 2007.

[12] _ - "Application of analytical retarded-time potential expressions to the solution of time domain integral equations," IEEE Trans. Antennas. Propag., vol. 59, no. 11, pp. 4123-4131, Nov. 2011.

[13] G. Vecchi, "Loop-star decomposition of basis functions in the discretization of the EFIE," IEEE Trans. Antennas. Propag., vol. 47, no. 2, pp. 339-346, Feb. 1999.

[14] F. P. Andriulli, K. Cools, H. Bagci, F. Olyslager, A. Buffa, S. Christiansen, and E. Michielssen, "A multiplicative Calderon preconditioner for the electric field integral equation," IEEE Trans. Antennas. Propag., vol. 56, no. 8, pp. 2398-2412, Aug. 2008.

[15] S. Y. Chen, W. C. Chew, J. M. Song, and J.-S. Zhao, "Analysis of low frequency scattering from penetrable scatterers," IEEE Trans. Geosci. Remote Sens., vol. 39, no. 4, pp. 726-735, Apr. 2001. 